Stud. Univ. Babeş-Bolyai Math. 64(2019), No. 1, 11-23

DOI: 10.24193/subbmath.2019.1.02

\title{
A topological representation of double Boolean lattices
}

\author{
Brigitte E. Breckner and Christian Săcărea
}

\begin{abstract}
Boolean Concept Logic has been introduced by R. Wille as a mathematical theory based on Formal Concept Analysis. Concept lattices are extended with two new operations, negation and opposition which then lead to algebras of protoconcepts which are equationally equivalent to double Boolean algebras. In this paper, we provide a topological representation for double Boolean algebras based on the so-called DB-topological contexts. A double Boolean algebra is then represented as the algebra of clopen protoconcepts of some DB-topological context.
\end{abstract}

Mathematics Subject Classification (2010): 18B35, 54B30, 68T30.

Keywords: Formal concept analysis, double boolean algebra, topological context.

\section{Introduction}

Formal Concept Analysis (FCA) is a prominent field of Applied Mathematics which is grounded on the mathematization of the notion of concept and concept hierarchy, having a wide range of applications in data analysis and knowledge discovery in databases. Topological FCA is an extension of FCA to topological spaces and is investigating issues related to the interplay between Topology and FCA.

Topological contexts were defined as an attempt to represent 0-1-lattices by open concepts of some topological context, i.e., formal concepts whose extents and intents are open sets. This theory was then completed to a categorical duality in a series of papers at the beginning of the 1990s, but for the sake of a more natural description, closed concepts were considered in order to represent 0-1-lattices ([3], [4]). Later on, a duality theory for 0-1 polarity lattices was developed in [5].

Categorical aspects in topological FCA have been studied in [1], especially for the metric case, while uniform contexts have been investigated in [8].

Bounded lattices, i.e., 0-1-lattices, have already been described by some topological representations by Stone [10], Priestley [7], and Urquhart [11]. Each of these representations is given via special topological spaces: compact totally disconnected spaces 
for Boolean lattices; spectral spaces or compact totally order-disconnected spaces for bounded distributive lattices; and the so-called $L$-spaces for arbitrary bounded lattices. In fact, when representing 0-1-lattices by standard topological contexts, one can recover all the above representations within the so-called double arrow space, i.e., a structure within the non-incidence of some topological context $\mathbb{K}^{\mathcal{T}}$.

Contextual Logic has been introduced by R. Wille as a logical extension of FCA "... with the aim to support knowledge representation and knowledge processing [...]. It is grounded on the traditional philosophical understanding of logic as the doctrine of the forms of thinking [...]" ([13].) While a logical extension of a formal context is quite straightforward, the same extension on concept lattices (which could be understood as the pattern counterpart of a context) is no longer straightforward, because of the semantics of the negation operator.

For introducing negations, two approaches were considered: a generalization of formal concepts to semiconcepts and protoconcepts, introducing the algebra of semiconcepts and the algebra of protoconcepts, respectively, which then leads to the notion of double Boolean algebra [6].

The main results of this paper are based on the representation theorem for double Boolean algebras from [6]. We define the notion of a DB-topological context in order to represent every double Boolean algebra as an algebra of clopen protoconcepts of a DB-topological context and show how this representation can be extended to a categorical duality.

\section{Formal Concept Analysis}

The basic structure FCA is using is a formal context. Using concept forming operators, formal concepts are extracted described as maximal patterns of incidences of a given binary relation. Concepts are ordered by the subconcept-superconcept relation and they form a conceptual hierarchy, i.e., a complete lattice which contains all knowledge patterns we can extract from a formal context. In Contextual Logic, a formal context is the mathematical structure in which the semantics of logical operators is declared, while for Conceptual Logic the same role is played by a concept lattice. Here we recall only some basic definitions. For more, we refer to [2].

Definition 2.1. A formal context is a triple $\mathbb{K}=(G, M, I)$, where $G$ and $M$ are sets and $I \subseteq G \times M$ is a binary relation. The set $G$ is called set of objects, $M$ is the set of attributes and $I$ is called incidence relation.

For sets $A \subseteq G$ and $B \subseteq M$, we define concept forming operators by $A^{\prime}=\{m \in M \mid g I m$ for all $g \in A\}$ and $B^{\prime}=\{g \in G \mid g I m$ for all $m \in B\}$. These operators form a Galois connection on the power sets of $G$ and $M$, respectively.

Definition 2.2. A formal concept of the context $\mathbb{K}=(G, M, I)$ is a pair $(A, B)$ with $A \subseteq G, B \subseteq M$ and $A^{\prime}=B, B^{\prime}=A$. The set $A$ is called extent and $B$ is called the intent of the concept $(A, B)$. The set of all concepts of $\mathbb{K}$ is denoted by $B(\mathbb{K})$.

On the set $B(\mathbb{K})$ of concepts we define the subconcept-superconcept relation by $\left(A_{1}, B_{1}\right) \leq\left(A_{2}, B_{2}\right) \Leftrightarrow A_{1} \subseteq A_{2}\left(\Leftrightarrow B_{1} \supseteq B_{2}\right)$. 
Theorem 2.3 (Basic Theorem on Concept Lattices). Let $\mathbb{K}:=(G, M, I)$ be a formal context. The concept lattice $(B(\mathbb{K}), \leq)$ is a complete lattice in which infimum and supremum are given by:

$$
\begin{aligned}
& \bigwedge_{t \in T}\left(A_{t}, B_{t}\right)=\left(\bigcap_{t \in T} A_{t},\left(\bigcup_{t \in T} B_{t}\right)^{\prime \prime}\right), \\
& \bigvee_{t \in T}\left(A_{t}, B_{t}\right)=\left(\left(\bigcup_{t \in T} A_{t}\right)^{\prime \prime}, \bigcap_{t \in T} B_{t}\right) .
\end{aligned}
$$

A complete lattice $V$ is isomorphic to $B(\mathbb{K})$ if and only if there are mappings $\tilde{\gamma}: G \rightarrow V$ and $\tilde{\mu}: M \rightarrow V$ such that $\tilde{\gamma}(G)$ is supremum-dense in $V, \tilde{\mu}(M)$ is infimum-dense in $V$ and $g I m$ is equivalent to $\tilde{\gamma} g \leq \tilde{\mu} m$ for all $g \in G$ and all $m \in M$. In particular, $V \simeq B(V, V, \leq)$.

Every object and every attribute can be recovered in the concept lattice of the given context. For an object $g \in G$, we write $g^{\prime}$ instead of $\{g\}^{\prime}$ for the object intent $\{m \in M \mid g I m\}$ of the object $g$. Correspondingly, $m^{\prime}$ stands for the attribute extent $\{g \in G \mid g I m\}$ of the attribute $m$. Using the symbols from the Basic Theorem, we write $\gamma g$ for the object concept $\left(g^{\prime \prime}, g^{\prime}\right)$ and $\mu m$ for the attribute concept $\left(m^{\prime}, m^{\prime \prime}\right)$.

Definition 2.4. A context $(G, M, I)$ is called clarified, if for any objects $g, h \in G$ from $g^{\prime}=h^{\prime}$ always follows that $g=h$ and, correspondingly, $m^{\prime}=n^{\prime}$ implies $m=n$ for all $m, n \in M$.

Definition 2.5. A clarified context $(G, M, I)$ is called row reduced, if every object concept is $\bigvee$-irreducible, and column reduced, if every attribute concept is $\wedge$-irreducible. A context which is row reduced and column reduced is called reduced.

If $(G, M, I)$ is a context, $g \in G$ an object, and $m \in M$ an attribute, we write

$$
\begin{aligned}
& g \swarrow m: \Leftrightarrow\left\{\begin{array}{c}
g\lfloor m \text { and } \\
\text { if } g^{\prime} \subseteq h^{\prime} \text { and } g^{\prime} \neq h^{\prime}, \text { then } h I m ;
\end{array}\right. \\
& g \nearrow m: \Leftrightarrow\left\{\begin{array}{c}
g\lfloor m \text { and } \\
\text { if } m^{\prime} \subseteq n^{\prime} \text { and } m^{\prime} \neq n^{\prime}, \text { then } g I n ;
\end{array}\right. \\
& g \measuredangle m: \Leftrightarrow g \ltimes m \text { and } g \nearrow m .
\end{aligned}
$$

Thus, $g \ltimes m$ if and only if $g^{\prime}$ is maximal among all object intents which do not contain $m$. In other words: $g \measuredangle m$ holds if and only if $g$ does not have the attribute $m$, but $m$ is contained in the intent of every proper subconcept of $\gamma g$.

The relation $\neg \subseteq G \times M$ is called the double-arrow space of the context $(G, M, I)$.

\section{Generalization of Concepts: Semi- and Protoconcepts}

As stated in [13] and [14], the research in the field of Contextual Logic is structured in three major themes: Contextual Concept Logic, Contextual Judgement Logic, and Contextual Conclusion Logic. In order to develop a satisfactory theory of Contextual Concept Logic, there was necessary to introduce a suitable notion of negation and to overcome some difficulties due to the fact that the complement of an extent 
(intent) is generally not an extent (intent). The solution was a generalization of concepts to semiconcepts and protoconcepts. While formal concepts are structured in a complete lattice, protoconcepts will give rise to a structure called double Boolean algebra.

In the following, we give the basic facts and results about algebras of protoconcepts and double Boolean algebras, for a complete information see [6] and [13].

Definition 3.1. Let $\mathbb{K}:=(G, M, I)$ be a formal context. A semiconcept of $\mathbb{K}$ is defined as a pair $(A, B)$ with $A \subseteq G$ and $B \subseteq M$ such that $A^{\prime}=B$ or $B^{\prime}=A$. A pair $(A, B)$ is called a protoconcept if $A^{\prime \prime}=B^{\prime}$ (or equivalently $A^{\prime}=B^{\prime \prime}$ ). We denote by $\mathfrak{H}(\mathbb{K})$ the set of all semiconcepts of a formal context $\mathbb{K}$. The set of all protoconcepts of $\mathbb{K}$ is denoted by $\mathfrak{P}(\mathbb{K})$. Obviously, every semiconcept is a protoconcept, hence $\mathfrak{H}(\mathbb{K}) \subseteq \mathfrak{P}(\mathbb{K})$.

In the following, we consider mainly sets of protoconcepts, pointing out the differences when semiconcepts are involved.

The set $\mathfrak{P}(\mathbb{K})$ of all protoconcepts of $\mathbb{K}$ carries a natural order $\subseteq$ which is defined by $\left(A_{1}, B_{1}\right) \subseteq\left(A_{2}, B_{2}\right): \Leftrightarrow A_{1} \subseteq A_{2}$ and $B_{1} \supseteq B_{2}$. This order does not generally yield a lattice structure on $\mathfrak{P}(\mathbb{K})$. However, there are natural operations on $\mathfrak{P}(\mathbb{K})$ which can be defined as follows:

$$
\begin{aligned}
\left(A_{1}, B_{1}\right) \sqcap\left(A_{2}, B_{2}\right) & :=\left(A_{1} \cap A_{2},\left(A_{1} \cap A_{2}\right)^{\prime}\right) \\
\left(A_{1}, B_{1}\right) \sqcup\left(A_{2}, B_{2}\right) & :=\left(\left(B_{1} \cap B_{2}\right)^{\prime}, B_{1} \cap B_{2}\right) \\
\neg(A, B) & :=\left(G \backslash A,(G \backslash A)^{\prime}\right) \\
\lrcorner(A, B) & :=\left((M \backslash B)^{\prime}, M \backslash B\right) \\
\perp & :=(\varnothing, M) \\
\top & :=(G, \varnothing) .
\end{aligned}
$$

The set $\mathfrak{P}(\mathbb{K})$ together with the operations $\sqcap, \sqcup\urcorner,\lrcorner, \perp$, and $T$ is called the algebra of protoconcepts of $\mathbb{K}$ and is denoted by $\mathfrak{P}(\mathbb{K})$. The operations are named meet, join, negation, opposition, nothing and all. The corresponding structure in the case of semiconcepts is the algebra of semiconcepts which will be denoted by $\underline{\mathfrak{H}}(\mathbb{K})$.

For an arbitrary element $x$ in $\mathfrak{P}(\mathbb{K})$ we denote by $x_{\sqcup}:=x \sqcup x$ and by $x_{\sqcap}:=x \sqcap x$. Let $\mathfrak{P}(\mathbb{K})_{\sqcap}:=\left\{\left(A, A^{\prime}\right) \mid A \subseteq G\right\}=\mathfrak{H}(\mathbb{K})_{\sqcap}$ and $\mathfrak{P}(\mathbb{K})_{\sqcup}:=\left\{\left(B^{\prime}, B\right) \mid B \subseteq M\right\}=\mathfrak{H}(\mathbb{K})_{\sqcup}$. Until now, there is no obvious difference between algebras of semiconcepts and algebras of protoconcepts. But if we have a closer look to their structure, from the definition of semiconcepts, we have $\mathfrak{H}(\mathbb{K})=\mathfrak{H}(\mathbb{K})_{\sqcap} \cup \mathfrak{H}(\mathbb{K})_{\sqcup}$, relation which does not hold for an algebra of protoconcepts. The main difference between algebras of semiconcepts and algebras of protoconcepts consists exactly in the existence of some "non-Boolean" elements whose influence on the structure and behaviour of such algebras is described in [6] and [13].

The formal concepts can be recovered in the meet of the two Boolean parts of $\mathfrak{P}(\mathbb{K})$, i.e., $\mathfrak{B}(\mathbb{K}):=\mathfrak{P}(\mathbb{K})_{\sqcap} \cap \mathfrak{P}(\mathbb{K})_{\sqcup}=\mathfrak{H}(\mathbb{K})_{\sqcap} \cap \mathfrak{H}(\mathbb{K})_{\sqcup}$, where $\mathfrak{B}(\mathbb{K})$ is the complete lattice of the (formal) concepts of $\mathbb{K}$. The term "non-Boolean" elements, respectively 
Boolean part of an algebra of protoconcepts (semiconcepts), is justified by the following additional operations defined as

$$
\begin{gathered}
x \uplus y:=\neg(\neg x \sqcap \neg y) \text { and } x \sqcap y:=\lrcorner(\lrcorner x \sqcup\lrcorner y), \\
\top:=\urcorner \perp \text { and } \perp:=\lrcorner \top .
\end{gathered}
$$

Obviously, $\mathfrak{P}(\mathbb{K})_{\sqcap}$ together with the restrictions of the operations $\sqcap, \varpi, \neg, \perp, \top$ is a Boolean algebra denoted $\mathfrak{P}(\mathbb{K})_{\sqcap}$, which is isomorphic to the Boolean algebra of all subsets of $G$. Dually, $\mathfrak{P}(\overline{\mathbb{K}}) \sqcup$ together with the restrictions of the operations $\Pi, \sqcup,\lrcorner, \perp, \top$ is a Boolean algebra denoted by $\underline{\mathfrak{P}}(\mathbb{K})_{\sqcup}$, and which is antiisomorphic to the Boolean algebra of all subsets of $M$.

Proposition 3.2. The following equations are valid in $\mathfrak{P}(\mathbb{K})$ :
1a) $(x \sqcap x) \sqcap y=x \sqcap y$
1b) $(x \sqcup x) \sqcup y=x \sqcup y$
2a) $x \sqcap y=y \sqcap x$
2b) $x \sqcup y=y \sqcup x$
3a) $x \sqcap(y \sqcap z)=(x \sqcap y) \sqcap z$
3b) $x \sqcup(y \sqcup z)=(x \sqcup y) \sqcup z$
4a) $x \sqcap(x \sqcup y)=x \sqcap x$
4b) $x \sqcup(x \sqcap y)=x \sqcup x$
5a) $x \sqcap(x \amalg y)=x \sqcap x$
5b) $x \sqcup(x \sqcap y)=x \sqcup y$
6a) $x \sqcap(x \amalg y)=(x \sqcap y) \amalg(x \sqcap z)$
6b) $x \sqcup(y \sqcap z)=(x \sqcup y)$ П $(x \sqcup z)$
7a) $\urcorner \neg(x \sqcap y)=x \sqcap y$
7b) $\lrcorner\lrcorner(x \sqcup y)=x \sqcup y$
8a) $\urcorner(x \sqcap x)=\urcorner x$
8b) $\lrcorner(x \sqcup x)=\lrcorner x$
9a) $x \sqcap\urcorner x=\perp$
9b) $x \sqcup\lrcorner x=\top$
10a) $\neg \perp=\mathrm{T} \sqcap \mathrm{T}$
10b) $\lrcorner \mathrm{T}=\perp \sqcup \perp$
11a) $\neg \mathrm{T}=\perp$
11b) $\lrcorner \perp=\mathrm{T}$
12) $(x \sqcap x) \sqcup(x \sqcap x)=(x \sqcup x) \sqcap(x \sqcup x)$.

Furthermore, the following condition holds in $\underline{\mathfrak{H}}(\mathbb{K})$ :

$$
\text { 13) } x=x \sqcap x \text { or } x=x \sqcup x \text {. }
$$

\section{Double Boolean Algebras}

Double Boolean algebras are algebraic structures $\underline{D}:=(D, \sqcap, \sqcup, \neg\lrcorner,, \perp, \top)$ of type $(2,2,1,1,0,0)$ satisfying the equations 1a) to $11 \mathrm{a}), 1 \mathrm{~b})$ to $11 \mathrm{~b})$ and 12$)$ of the precedent Proposition. If a double Boolean algebra satisfies also condition 13) of Proposition 3.3 .1 , it is called pure. An algebraic structure of the type $(2,2,1,1,0,0)$ in which only equations 1a) to 11a) and 1b) to 11b) are valid is called weak double Boolean algebra. As one can easily see, algebras of protoconcepts are double Boolean algebras, while algebras of semiconcepts are pure double Boolean algebras.

We can define a quasiorder on a (weak) double Boolean algebra similar to that defined on the algebra of protoconcepts, namely

$$
x \sqsubseteq y: \Leftrightarrow x \sqcap y=x \sqcap x \text { and } x \sqcup y=y \sqcup y .
$$

Lemma 4.1. [13] In a (weak) double Boolean algebra the following conditions hold:

(1) $x \sqcap y \sqsubseteq x \sqsubseteq x \sqcup y$,

(2) the mapping $x \mapsto x \sqcap y$ preserves $\sqsubseteq$ and $\sqcap$,

(3) the mapping $x \mapsto x \sqcup y$ preserves $\sqsubseteq$ and $\sqcup$. 
For a (weak) double Boolean algebra $\underline{D}:=(D, \sqcap, \sqcup\urcorner,\lrcorner, \perp, \top)$ further operations are defined as in the preceding section by

$$
\begin{gathered}
x \uplus y:=\urcorner(\neg x \sqcap y) \text { and } x \text { 吊 } y:=\lrcorner(\lrcorner x \sqcup\lrcorner y), \\
\top:=\urcorner \perp \text { and } \underline{\perp}:=\lrcorner \top .
\end{gathered}
$$

In addition, let $x_{\sqcap}:=x \sqcap x$ and $x_{\sqcup}:=x \sqcup x$. Lead again by the preceding section we define $D_{\sqcap}:=\left\{x_{\sqcap} \mid x \in D\right\}$ and $D_{\sqcup}:=\left\{x_{\sqcup} \mid x \in D\right\}$. Now, (weak) double Boolean algebras can be characterized as special ordered structures.

Proposition 4.2. [6] Let $\underline{D}:=(D, \sqcap, \sqcup\urcorner,\lrcorner, \perp, \top)$ be a (weak) double Boolean algebra. Then the following conditions are satisfied.

(1) $(D$, ㄷ) is a quasi-ordered set.

(2) $\left.\underline{D}_{\sqcap}:=\left(D_{\sqcap}, \sqcap, \uplus,\right\urcorner, \perp, \top\right)$ is a Boolean algebra whose order relation is the restriction of 5 to $D_{\sqcap}$.

(3) $\left.\underline{D}_{\sqcup}:=\left(D_{\sqcup}, \Pi, \sqcup,\right\lrcorner, \perp, \top\right)$ is a Boolean algebra whose order relation is the restriction of $\sqsubseteq$ to $D_{\sqcup}$.

(4) $y \sqsubseteq x_{\sqcap} \Leftrightarrow y \sqsubseteq x$ for $x \in D$ and $y \in D_{\sqcap}$.

(5) $x_{\sqcup} \sqsubseteq y \Leftrightarrow x \sqsubseteq y$ for $x \in D$ and $y \in D_{\sqcup}$.

(6) $x \sqsubseteq y \Leftrightarrow x_{\sqcap} \sqsubseteq y_{\sqcap}$ and $x_{\sqcup} \sqsubseteq y_{\sqcup}$ for $x, y \in D$.

How close weak double Boolean algebras are to double Boolean algebras is made clear by the following Proposition.

Proposition 4.3. [6] Let $\underline{D}:=(D, \sqcap, \sqcup, \neg\lrcorner,, \perp, \top)$ be a weak double Boolean algebra with $D=D_{\sqcap} \cup D_{\sqcup}$. Then $\underline{\text { ㅁ }}:=\{(x, x) \mid x \in D\} \cup\left\{\left(x_{\sqcap \sqcup}, x_{\sqcup \sqcap}\right) \mid x \in D\right\} \cup\left\{\left(x_{\sqcup \sqcap}, x_{\sqcap \sqcup}\right) \mid x \in D\right\}$ is a congruence relation of $\underline{D}$.

\section{Filters of Double Boolean Algebras}

Let $\underline{D}$ be a double Boolean algebra. Our task is to give a topological representation of a double Boolean algebra as an algebra of protoconcepts of a suitable topological context. First, we have to remark that algebras of protoconcepts are ordered structures, so we will consider only regular double Boolean algebras, i.e., double Boolean algebras for which $\subseteq$ is an order. There is no restriction of generality since every double Boolean algebra can be regularized by a suitable factorization.

Definition 5.1. Let $\underline{L}$ be an ordered set, $F$ be a filter of $\underline{L}$, and $I$ an ideal of $\underline{L}$. We say that $F$ is $I$-maximal if $F$ is a maximal filter which is disjoint from $I$. Dually, $F$-maximal ideals are defined. We obtain the following sets:

$$
\begin{aligned}
\mathfrak{F}_{0}(\underline{L}) & :=\{F \subseteq L \mid \exists I \in \mathfrak{I}(\underline{L}): F \text { is } I \text { maximal }\} \\
\mathfrak{I}_{0}(\underline{L}) & :=\{I \subseteq L \mid \exists I \in \mathfrak{F}(\underline{L}): I \text { is } F-\text { maximal }\} \\
\mathfrak{M}(\underline{L}) & :=\left\{(F, I) \in \mathfrak{F}_{0}(\underline{L}) \times \mathfrak{I}_{0}(\underline{L}) \mid F \text { is } I-\right.\text { maximal and } \\
& I \text { is } F \text {-maximal }\} .
\end{aligned}
$$

The elements of $\mathfrak{M}(\underline{L})$ are called maximal filter-ideal pairs.

Definition 5.2. A filter of a double Boolean algebra $\underline{D}$ is defined to be to be a subset $F$ of $D$ satisfying 
1. $x \in F$ and $x \sqsubseteq y$ in $D$ imply $y \in F$;

2. If $x \in F$ and $y \in F$ then $x \sqcap y \in F$.

An ideal of $\underline{D}$ is defined dually. A subset $F_{0}$ is called a base of a filter $F$ if $F=\{y \in$ $D \mid x$ ᄃ $y$ for some $\left.x \in F_{0}\right\}$, and we write $F=\uparrow F_{0}$. A base of an ideal is defined dually. If $\underline{D}$ is a regular double Boolean algebra, we denote by $\mathfrak{F}(\underline{D})$ the set of all filters of $\underline{D}$ and by $\mathfrak{I}(\underline{D})$ the set of all ideals of $\underline{D}$. A filter $F$ of $\underline{D}$ is called prime if $F \cap \underline{D}$ is a prime filter in $D_{\sqcap}$; a prime ideal is defined dually. The prime filters and prime ideals are collected in $\mathfrak{F}_{p}(\underline{D})$ and $\mathfrak{I}_{p}(\underline{D})$, respectively.

Lemma 5.3. The following holds true:

1. $F$ is a filter of a regular double Boolean algebra $\underline{D}$ if and only if the characteristic function is an order homomorphism preserving $\sqcap$.

2. The filter $F$ is prime if and only if the characteristic function of $F$ preservers $\urcorner$ too.

Proof. $\quad$ 1. Let $F \in \mathfrak{F}(D)$. We define the map $\phi: \underline{D} \rightarrow \underline{2}$ by

$$
\phi(x)= \begin{cases}1, & x \in F \\ 0, & x \notin F .\end{cases}
$$

Let $x, y \in F$, then $x \sqcap y \in F$ and so $\phi(x \sqcap y)=\phi(x) \wedge \phi(y)=1$. For $x \in F$ and $y \notin F$ or $x \notin F$ and $y \in F$ we have that $x \sqcap y \notin F$, hence $\phi(x \sqcap y)=\phi(x) \wedge \phi(y)=0$.

Now, for $x, y \notin F$, we have that $x \sqcap y \notin F$ and, since $T \in F$, we obtain that $\phi$ is a $\sqcap$-homomorphism. Define $F:=\phi^{-1}(1)$, then $\phi(x)=\phi(y)=1$ for $x, y \in F$ and so $\phi(x \sqcap y)=1$, implying that $x \sqcap y \in F$. Let now $x \in F$ and $y \in D$ with $x$ 드. Then, by definition, $\phi(x)=1$ and

$$
x \sqsubseteq y \Leftrightarrow x \sqcap y=x \sqcap x \text { and } x \sqcup y=y \sqcup y ;
$$

hence $\phi(x \sqcap y)=\phi(x) \wedge \phi(y)=\phi\left(x_{\sqcap}\right)$. Since $x \in F$, we have $\phi\left(x_{\sqcap}\right)=1$. By Lemma 4.1, from $x \sqsubseteq y$ follows $x \sqcap y \sqsubseteq y \sqcap y$, and since $\phi$ was an order homomorphism, we have that $\phi\left(y_{\sqcap}\right)=1$, i.e., $y_{\sqcap} \in F$. We conclude that $F_{\sqcap}$ is a filter of $\underline{D}_{\square}$, hence $F$ is a filter of $\underline{D}$ too.

2. Let $F$ be a prime filter and $\phi: \underline{D} \rightarrow \underline{2}$ defined as above. As one can easily see, the only case where a proof deserves to be made is $x, y \notin F$. Consider $x, y \notin F$ arbitrary chosen, then $x_{\sqcap}, y_{\sqcap} \notin F$. But $F \cap \underline{D}_{\sqcap}$ is a prime filter of the Boolean algebra $\underline{D}_{\sqcap}$, hence $\urcorner x_{\sqcap}, \neg y_{\sqcap} \in F \cap \underline{D}_{\sqcap}$. It follows that $\left.\phi\left(\neg x_{\sqcap}\right)=\phi(\neg x)=\right\urcorner \phi(x)$. For $x \in F$, we obviously have $\phi(\neg x)=\urcorner \phi(x)$; hence $\phi$ is a $\urcorner$ - homomorphism, since the same holds for $x \notin F$.

Remark 5.4. If $F \in \mathfrak{F}_{p}(\underline{D})$, then $F \cap \underline{D}_{\Pi}$ is a prime filter of the Boolean algebra $\underline{D}_{\Pi}$ and so there is an ideal $I$ of $\underline{D}_{\sqcap}$, so that $F \cap \underline{D}_{\sqcap}$ is $I$-maximal and dually for prime ideals.

Let now $F$ be a filter in $\underline{D}$ and $x \in F$. Then $x \sqcap x=: x_{\sqcap} \in F$ and so $F \cap \underline{D}_{\sqcap} \neq \varnothing$. For any $x_{\sqcap} \in F$ we have that $x \in F$ since $x \sqcap x$ 드. It follows that $x \in F \Leftrightarrow x_{\sqcap} \in F$. Hence $F \cap \underline{D}_{\sqcap} \in \mathfrak{F}\left(\underline{D}_{\sqcap}\right)$. In fact, $x_{\sqcap} \in F$ and $x_{\sqcap} \subseteq y_{\sqcap}$ implies $y_{\sqcap} \in F$ and so $y_{\sqcap} \in F \cap \underline{D}_{\Pi}$.

An easy calculation shows the validity of the following Lemma: 
Lemma 5.5. Let $L$ and $M$ be two regular double Boolean algebras and $f: L \rightarrow M$ an onto homomorphism. Then

a) $F \in \mathfrak{F}(L) \Rightarrow f(F) \in \mathfrak{F}(M)$, and $I \in \mathfrak{I}(L) \Rightarrow f(I) \in \mathfrak{I}(M)$.

b) $E \in \mathfrak{F}_{p}(M) \Rightarrow f^{-1}(E) \in \mathfrak{F}_{p}(L)$ and $H \in \mathfrak{I}_{p}(M) \Rightarrow f^{-1}(H) \in \mathfrak{I}_{p}(L)$.

c) $(E, H) \in \mathfrak{M}(M) \Rightarrow\left(f^{-1}(E), f^{-1}(H)\right) \in \mathfrak{M}(L)$.

We conclude this section with a basic result from [6].

Lemma 5.6. Let $F$ be a filter of a double Boolean algebra $\underline{D}$.

1. $F \cap D_{\sqcap}$ and $F \cap D_{\sqcup}$ are filters of the Boolean algebra $\underline{D}_{\sqcap}$ and $\underline{D}_{\sqcup}$, respectively;

2. Each filter of the Boolean algebra $\underline{D}_{\Pi}$ is a base of some filter of $\underline{D}$; in particular, $F \cap D_{\sqcap}$ is a base of $F$.

\section{Topological Representation}

Double Boolean algebras play a substantial role in the development of the Concept Logic, a role which is in a certain way similar to that played by Boolean algebras in the classical Logic. In the following, we develop a topological representation theory for regular double Boolean algebras, i.e., double Boolean algebras for which the quasiorder $\subseteq$ is an order relation.

A topological space is denoted by $(X, \mathcal{T})$ where $\mathcal{T}$ is the set of all closed subsets of $X$. Consider the context $\mathbb{K}:=(G, M, I)$ and let $\mathcal{T}$ be a topology on $G$ and $\tau$ be a topology on $M$. A clopen protoconcept is a pair $(A, B) \in \mathfrak{P}(\mathbb{K})$ with $A \subseteq G$ clopen, and $B \subseteq M$ clopen too. We denote the set of all clopen protoconcepts by $\mathfrak{P}^{c o}((G, \mathcal{T}),(M, \tau), I)$.

Definition 6.1. $\mathbb{K}^{D B}:=((G, \mathcal{T}),(M, \tau), I)$ is called a DB-topological context if:

(i) $(G, \mathcal{T})$ and $(M, \tau)$ are topological spaces and $I \subseteq G \times M$.

(ii) If $A \subseteq G$ is a clopen set then $A^{\prime} \subseteq M$ is clopen too, and the same holds for clopen sets in $M$.

(iii) A subbasis for the closed and for the open sets in $G$ is given by the extents of clopen protoconcepts of $\mathbb{K}^{D B}$ and, dually, a subbasis for the closed and for the open subsets of $M$ is given by the intents of clopen protoconcepts of $\mathbb{K}^{D B}$.

Remark 6.2. $\quad$ 1. The set $\mathfrak{P}^{c o}\left(\mathbb{K}^{D B}\right)$ inherits the ordering from $\underline{\mathfrak{P}}\left(\mathbb{K}^{D B}\right)$. We shall denote this ordered set by $\mathfrak{P}^{c o}\left(\mathbb{K}^{D B}\right)$.

2. Using the same idea as in [3], we are able to represent the 0-1-lattice $\underline{D}_{\sqcap} \cap \underline{D}_{\sqcup}$ by the 0-1-lattice of clopen concepts of a suitable DB-topological context.

Remark 6.3. $\mathfrak{P}^{c o}\left(\mathbb{K}^{D B}\right)$ is a subalgebra of $\mathfrak{P}\left(\mathbb{K}^{D B}\right)$. Moreover, it becomes now evident that it is necessary to consider clopen protoconcepts since the negation of a closed protoconcept would not be any longer a closed protoconcept.

Proposition 6.4. For every DB-topological context $\mathbb{K}^{D B}$, the ordered set of clopen protoconcepts $\underline{\mathfrak{P}}^{c o}\left(\mathbb{K}^{D B}\right)$ is a regular double Boolean algebra. 
Proof. Remember that if $(A, B)$ and $(C, D)$ are clopen protoconcepts, then

$$
\begin{aligned}
(A, B) \sqcap(C, D) & =\left(A \cap C,(A \cap C)^{\prime}\right) \\
(A, B) \sqcup(C, D) & =\left((B \cap D)^{\prime}, B \cap D\right) \\
\neg(A, B) & =\left(G \backslash A,(G \backslash A)^{\prime}\right) \\
\lrcorner(A, B) & =\left((M \backslash B)^{\prime}, M \backslash B\right) \\
\perp & =(\varnothing, M) \\
\mathrm{\top} & =(G, \varnothing) .
\end{aligned}
$$

Since the involved sets are all clopen, it follows that the restrictions of these operations to $\mathfrak{P}^{c o}\left(\mathbb{K}^{D B}\right)$, i.e., meet, join, negation, opposition, nothing and all are well-defined. Since the ordering on $\mathfrak{P}^{c o}\left(\mathbb{K}^{D B}\right)$ is that inherited from $\mathfrak{P}\left(\mathbb{K}^{D B}\right)$, it follows that $\underline{\mathfrak{P}}^{c o}\left(\mathbb{K}^{D B}\right)$ is a regular double Boolean algebra.

For a regular double Boolean algebra $\underline{D}$, we define the standard context of $\underline{D}$ as $\mathbb{K}(\underline{D}):=\left(\mathfrak{F}_{p}(\underline{D}), \mathfrak{I}_{p}(\underline{D}), \Delta\right)$, where $F \Delta I: \Leftrightarrow F \cap I \neq \varnothing$. On $\mathfrak{F}_{p}(\underline{D})$ consider the topology generated by the subbasis $\left\{F_{x} \mid x \in \underline{D}\right\}$ with $F_{x}:=\left\{F \in \mathfrak{F}_{p}(\underline{D}) \mid x \in F\right\}$. Dually, on $\mathfrak{I}_{p}(\underline{D})$ consider the topology generated by the subbasis $\left\{I_{x} \mid x \in \underline{D}\right\}$ with $I_{x}:=\left\{I \in \mathfrak{I}_{p}(\underline{D}) \mid x \in I\right\}$. We denote the context $\mathbb{K}(\underline{D})$ with the above topologies by $\mathbb{K}^{D B}(\underline{D})$ and we will prove that $\mathbb{K}^{D B}(\underline{D})$ is a DB-topological context.

Lemma 6.5. [6] The derivations in $\mathbb{K}^{D B}(\underline{D})$ yield:

1. $F_{x}^{\prime}=I_{x}=I_{x_{\sqcup}}$ for all $x \in D_{\sqcap}$.

2. $I_{y}^{\prime}=F_{y}=F_{y_{\Pi}}$ for all $y \in D_{\sqcup}$.

3. $F_{z}^{\prime}=I_{z_{\sqcap}}=I_{z_{\Pi \sqcup}}$ and $I_{z}^{\prime}=F_{z_{\sqcup}}=F_{z_{\sqcup}}$ for all $z \in D \backslash\left(D_{\sqcap} \cap D_{\sqcup}\right)$.

The following result is true:

Theorem 6.6. For every regular double Boolean algebra $\underline{D}$, the context $\mathbb{K}^{D B}(\underline{D})$ is a topological context.

Proof. We first remark that, by Lemma 6.5 , the pair $\left(F_{x}, I_{x}\right)$ is a protoconcept of $\underline{\mathfrak{P}}^{c o}\left(\mathbb{K}^{D B}(\underline{D})\right)$ for every $x \in \underline{D}$ : For any $x \in \underline{D}_{\sqcap}$ we have $F_{x}^{\prime}=I_{x}=I_{x_{\sqcup}}$ and so $\bar{F}_{x}^{\prime \prime}=I_{x_{\sqcup}}^{\prime}=F_{x_{\sqcup}}=F_{x_{\sqcup \Pi}}$ and $I_{x}^{\prime}=I_{x_{\sqcup}}^{\prime}=F_{x_{\sqcup}}=F_{x_{\sqcup \Pi}}$. The same holds for $x \in \underline{D}_{\sqcup}$ and for the "non-Boolean" elements in $D \backslash\left(\underline{D}_{\sqcap} \cap \underline{D}_{\sqcup}\right)$. Moreover, the complement of an element of the given subbasis, $c F_{x}=\mathfrak{F}_{p}(\underline{D}) \backslash F_{x}=\left\{F \in \mathfrak{F}_{p}(\underline{D}) \mid x \notin F\right\}=F_{\neg x}$, is again in that family, so every element of the subbasis is clopen. We want to prove now the second condition, namely that for a clopen $C \subseteq \mathfrak{F}_{p}(\underline{D})$, its derivation, $C^{\prime}$, is clopen too.

Let $C \subseteq \mathfrak{F}_{p}(\underline{D})$ be a clopen set, then $C$ is closed and

$$
C=\bigcap_{j \in J}\left(\bigcup_{a \in A_{j}} F_{a}\right)
$$

where $J \neq \varnothing$ is an arbitrary index-set and $A_{j} \subseteq D$ are finite sets for every $j \in J$.

Its complement

$$
c C=\bigcup_{j \in J}\left(\bigcap_{a \in A_{j}} c F_{a}\right)=\bigcup_{j \in J}\left(\bigcap_{a \in A_{j}} F_{\urcorner a}\right)
$$


can be expressed in terms of the given subbasis too, proving that the family $\left\{F_{x} \mid x \epsilon\right.$ $\underline{D}\}$ is also a subbasis for the open subsets of $\mathfrak{F}_{p}(\underline{D})$. Hence an arbitrary closed set $C \subseteq \mathfrak{F}_{p}(\underline{D})$ will be expressed as $C=\bigcap_{j \in J}\left(\cup_{a \in A_{j}} F_{a}\right)$ and we denote the set of all finite subsets of $J$ with $\mathcal{E}_{J}$. Then, for every $E \in \mathcal{E}_{J}$, the order ideal $\bigcap_{j \in E} \downarrow A_{j}$ has finitely many maximal elements. We denote the set of these maximal elements by max $\bigcap_{j \in E} \downarrow A_{j}$. Thus, the element

$$
s_{E}:=\bigsqcup \max \bigcap_{j \in E} \downarrow A_{j}
$$

is well-defined for every $E \in \mathcal{E}_{J}$ and belongs to $\underline{D}$. Define $B_{J}:=\left\{s_{E} \mid E \in \mathcal{E}_{J}\right\}$.

We claim that

$$
C^{\prime}=\bigcup_{a \in B_{J}} I_{a} .
$$

To prove this, let $a \in B_{J}$ and $I \in I_{a}$. It follows that there is an $E \in \mathcal{E}_{J}$ with $s_{E}=a$. The filter $F$ belongs to $C$ if and only if $F \cap A_{j} \neq \varnothing$ for all $j \in J$, and so $F \cap A_{j} \neq \varnothing$ for $j \in E$, concluding that $s_{E} \in F$. This statement is equivalent to $F \Delta I$ for all $F \in C$; hence $I \in C^{\prime}$.

Consider $I \in \mathfrak{I}_{p}(\underline{D})$ with $a \notin I$ for all $a \in B_{J}$. For all $E \in \mathcal{E}_{J}$, there is a map

$$
f_{E}: E \rightarrow \bigcup_{j \in J} A_{j}
$$

with $f_{E}(j) \in A_{j}$ and $\sqcap f_{E}(E) \notin I$, hence $s_{E} \in\left(\uparrow \sqcap f_{E}(E)\right) \backslash I$. (Choose for $f_{E}(j)$ one of the maximal elements of $\downarrow A_{j}$ which appears in $s_{E}$. Then $s_{E} \in \uparrow \sqcap f_{E}(E)$, but $s_{E} \notin I$.)

Rado's Selection Theorem yields the existence of a map $f: J \rightarrow \cup_{j \in J} A_{j}$ defined by $f(j) \in A_{j}$ for every $j \in J$, so that, for all $E \in \mathcal{E}_{J}$, there is a filter $F \in \mathcal{E}_{J}$ with $E \subseteq F$ and $f_{\left.\right|_{E}}=f_{\left.F\right|_{E}}$. It follows that the filter generated by $\{f(j) \mid j \in J\}$ contains $B_{J}$ and is disjoint from $I$. Zorn's Lemma guarantees the existence of an $I$-maximal filter containing the given one and so $I \notin C^{\prime}$. We just have proved the openness of the set $C^{\prime}$ in $\mathfrak{I}_{p}(\underline{D})$.

The set $C$ is clopen and the family $\left\{F_{x} \mid x \in \underline{D}\right\}$ is a subbasis for the open sets in $\mathfrak{F}_{p}(\underline{D})$; hence $C=\bigcup_{k \in K}\left(\bigcap_{b \in B_{k}} F_{b}\right)$, where $K \neq \varnothing$ is an index-set and $B_{k}$ are finite subsets of $D$ for every $k \in K$. Since

$$
\bigcap_{b \in B_{k}} F_{b}=\left\{F \in \mathfrak{F}_{p}(\underline{D}) \mid \forall b \in B_{k}: b \in F\right\}=F_{B_{k}},
$$

we have that $C=\bigcup_{k \in K} F_{B_{k}}=\bigcup_{k \in K} F_{B_{k}}$, where $B_{k \sqcap}:=\left\{b_{\sqcap} \mid b \in B_{k}\right\}$.

The following holds:

$$
C^{\prime}=\bigcap_{k \in K} F_{B_{k_{\Pi}}}^{\prime}=\bigcap_{k \in K} I_{B_{k_{\Pi}}}=\bigcap_{k \in K} I_{B_{k_{\Pi}}}=\bigcap_{k \in K} \bigcap_{b \in B_{k \sqcap \sqcup}} I_{b}
$$

concluding that $C^{\prime}$ is also closed and since it was also open, it is clopen.

Now, we are able to give a representation theorem for regular double Boolean algebras in terms of Formal Concept Analysis.

Theorem 6.7. Let $\underline{D}$ be a regular double Boolean algebra. Then

$$
\iota: \underline{D} \rightarrow \underline{\mathfrak{P}}^{c o}\left(\mathbb{K}^{D B}(\underline{D})\right), \quad a \mapsto\left(F_{a}, I_{a}\right)
$$

is an isomorphism. 
Proof. By Lemma 6.5, $\left(F_{x}, I_{x}\right)$ is a protoconcept of $\mathbb{K}(\underline{D})$ for all $x \in \underline{D}$. For $x \notin y$ in $\underline{D}_{\Pi}$, there is always an $F \in \mathfrak{F}_{p}(\underline{D})$ with $x \in F$ but $y \notin F$; hence $F_{x} \neq F_{y}$ and so $\left(F_{x}, I_{x}\right) \neq\left(F_{y}, I_{y}\right)$. Such inequality can be obtained dually for $y \nsucceq x$ in $\underline{D}_{\sqcup}$. If $x \nsubseteq y$ in $\underline{D}$ with $x \notin D_{\sqcup}$ and $y \notin D_{\sqcap}$ then, because $\uparrow x:=\{y \in D \mid x \sqsubseteq y\}$ is a filter of $\underline{D}_{\sqcup}$ by Lemma 4.1, there exists an $I \in \mathfrak{I}_{p}(\underline{D})$ with $x \in I$ but $y \notin I$; hence we have $\left(F_{x}, I_{x}\right) \neq\left(F_{y}, I_{y}\right)$ also in this case and its dual.

Using Theorem 2 from [13], we deduce that the map $\iota$ is a homomorphism, since $F_{x \sqcap y}=F_{x} \cap F_{y}, I_{x \sqcup y}=I_{x} \cap I_{y}, F_{\neg x}=\mathfrak{F}_{p}(\underline{D}) \backslash F_{x}$ and $I_{\lrcorner x}=\mathfrak{I}_{p}(\underline{D}) \backslash I_{x}$. These equalities result from the following equivalences and their duals: $F \in F_{x \sqcap y} \Leftrightarrow x \sqcap y \in F \Leftrightarrow x, y \in$ $F \Leftrightarrow F \in F_{x} \cap F_{y}$ and $F \in F_{\neg x} \Leftrightarrow \neg x \in F \Leftrightarrow \neg(x \sqcap x) \in F \Leftrightarrow x \sqcap x \notin F \Leftrightarrow x \notin F \Leftrightarrow F \in$ $\mathfrak{F}_{p}(\underline{D}) \backslash F_{x}$ (for a detailed proof of these equivalences, see [13]).

We want to prove that $\iota$ is onto. For this, let $(A, B) \in \mathfrak{P}^{c o}(\mathbb{K}(\underline{D}))$ be a clopen protoconcept, i.e., $A=\bigcap_{j \in J}\left(\bigcup_{a \in A_{j}} F_{a}\right)$ where $J$ is an index set and $A_{j}$ are finite subsets of $D$ for every $j \in J$. But $\cup_{a \in A_{j}} F_{a}$ is a member of the given subbasis, since $c\left(\cup_{a \in A_{j}} F_{a}\right)=\bigcap_{a \in A_{j}} c F_{a}=\bigcap_{a \in A_{j}} F_{\urcorner a}=F_{\urcorner A_{j}}=F_{\sqcap\urcorner A_{j}}$. The set $A_{j}$ is finite for every $j \in J$, so there is an $x_{j} \in D$ with $\left.x_{j}=\sqcap\right\urcorner A_{j}$; hence $\bigcup_{a \in A_{j}} F_{a}=F_{\urcorner x_{j}}$. By Lemma 2 [13], there is a filter $X \in \mathfrak{F}(\underline{D})$ with $A=\bigcap_{j \in J}\left(\bigcup_{a \in A_{j}} F_{a}\right)=F_{X}$.

Let us denote by $A_{\sqcap}:=\left\{F_{\Pi} \in \mathfrak{F}_{0}\left(\underline{D}_{\sqcap}\right) \mid F_{\Pi}=F \cap \underline{D}_{\Pi}, F \in A\right\}$. Since $A=$ $\bigcap_{j \in J}\left(\cup_{a \in A_{j}} F_{a}\right)$, we prove that $A_{\sqcap}=\bigcap_{j \in J}\left(\bigcup_{a_{\Pi} \in A_{j}}\left(F_{a_{\Pi}}\right)_{\Pi}\right)$. Let now $F_{\sqcap} \in A_{\Pi}$, then there exists a filter $F \in A$, with $F_{\sqcap}=F \cap \underline{D}_{\Pi}$. The following holds:

$$
\begin{aligned}
F \in A & \Leftrightarrow \forall j \in J \exists a \in A_{j}: a \in F \\
& \Rightarrow \forall j \in J \exists a_{\sqcap} \in A_{j_{\Pi}}: a_{\sqcap} \in F \\
& \Rightarrow F_{\sqcap} \in \bigcap_{j \in J}\left(\bigcup_{a_{\Pi} \in A_{j_{\Pi}}} F_{a_{\Pi}}\right) .
\end{aligned}
$$

For the second inclusion, the following holds

$$
\begin{aligned}
F_{\sqcap} & \in \bigcap_{j \in J}\left(\bigcup_{a_{\Pi} \in A_{j}} F_{a_{\Pi}}\right) \\
& \Rightarrow \forall j \in J \exists a_{\sqcap} \in A_{j_{\sqcap}}: a_{\sqcap} \in F_{\sqcap} \\
& \Rightarrow \forall j \in J \exists a_{\sqcap} \in A_{j_{\sqcap}}: a_{\sqcap} \in F:=\uparrow F_{\sqcap} \\
& \Rightarrow \exists F \in A: F_{\sqcap}=F \cap \underline{D}_{\sqcap} .
\end{aligned}
$$

This proves the closeness of $A_{\sqcap}$ in $\mathfrak{F}_{0}\left(\underline{D}_{\sqcap}\right)$. We have seen that $A_{\sqcap}=\left(F_{X_{\Pi}}\right)_{\sqcap}$. In order to prove that $A_{\sqcap}$ is a closed extent we use a similar approach to Proposition 5 from [3]. Hence there is an $a \in \underline{D}_{\sqcap}$ with $A_{\sqcap}=\left(F_{a_{\sqcap}}\right)_{\sqcap}$, i.e., $A=F_{a}$ for a suitable $a \in \underline{D}$, which completes the proof.

Remark 6.8. The topological representation of regular double Boolean algebras arises from the topological representation of bounded lattices, considering every filter as a basis of a filter $\mathfrak{P}^{c o}(\mathbb{K}(\underline{D}))$ and dually for ideals.

With these considerations, we are now able to develop a duality for regular double Boolean algebras. The following simple observations, will be used repeteadly.

Lemma 6.9. Consider $F \in \mathfrak{F}(\underline{D})$ and $I \in \mathfrak{I}(\underline{D})$.

1. If $F \cap I=\varnothing$, then there is an $\bar{F} \in \mathfrak{F}_{p}(\underline{D})$ with $F \subseteq \bar{F}$ and $\bar{F}$ is I-maximal. 
2. If $F \cap I=\varnothing$, then there is an $\bar{I} \in \mathfrak{I}_{p}(\underline{D})$ with $I \subseteq \bar{I}$ and $\bar{I}$ is $F$-maximal.

3. If $F \cap I=\varnothing$, then there is an $\bar{F} \in \mathfrak{F}_{p}(\underline{D})$ and an $\bar{I} \in \mathfrak{I}_{p}(\underline{D})$ with $F \subseteq \bar{F}$ and $I \subseteq \bar{I}$, so that $F$ is $I$-maximal and $I$ is $F$-maximal.

Remark 6.10. A filter $F \in \mathfrak{F}_{p}(\underline{D})$ if and only if there is an ideal $I \in \mathfrak{I}_{p}(\underline{D})$ such that $F$ is $I$-maximal.

Lemma 6.11. Let $\underline{D}$ be a regular double Boolean algebra, $F \in \mathfrak{F}_{p}(\underline{D})$ and $I \in \mathfrak{I}_{p}(\underline{D})$. Then:

1. $F$ is $I$-maximal $\Leftrightarrow F \ltimes I$ in $\mathbb{K}^{D B}(\underline{D})$.

2. I is $F$-maximal $\Leftrightarrow F \nearrow I$ in $\mathbb{K}^{D B}(\underline{D})$.

3. $(F, I) \in \mathfrak{M}(\underline{D}) \Leftrightarrow F \measuredangle I$ in $\mathbb{K}^{D B}(\underline{D})$.

Remark 6.12. By the previous Lemma, we observe that the context $\mathbb{K}^{D B}(\underline{D})$ is the context reduction of $(\mathfrak{F}(\underline{D}), \mathfrak{I}(\underline{D}), \Delta)$.

Definition 6.13. A DB-topological context is called standard if in addition the following hold:

(R) $\mathbb{K}^{D B}$ is reduced;

(S) $g I m \Rightarrow \exists(A, B) \in \mathfrak{P}^{c o}\left(\mathbb{K}^{D B}\right): g \in A$ and $m \in B$;

(Q) $\left(c I,(\rho \times \sigma)_{\mid c I}\right)$ is a quasicompact space where $c I:=(G \times M) \backslash I$ and $\rho \times \sigma$ denotes the product topology on $G \times M$.

Remark 6.14. The topological context of a regular double Boolean algebra $\mathbb{K}^{D B}(\underline{D})$ is standard, due to the analogy to standard topological contexts as they have been defined in [4].

The next Theorem completes our representation and its proof is similar to that given by G. Hartung in [4] to the main representation theorem for standard topological contexts. We only have to modify some sections were concepts have to be replaced by protoconcepts, but this is an easy and routine job.

Theorem 6.15. Let $\mathbb{K}^{D B}$ be a DB-standard topological context. The mappings

$$
\begin{gathered}
\alpha: G \rightarrow \mathfrak{F}_{p}\left(\underline{\mathfrak{P}}^{c o}\left(\mathbb{K}^{D B}\right)\right), \quad g \mapsto\left\{(A, B) \in \mathfrak{P}^{c o}\left(\mathbb{K}^{D B}\right) \mid g \in A\right\} \\
\beta: M \rightarrow \mathfrak{I}_{p}\left(\underline{\mathfrak{P}}^{c o}\left(\mathbb{K}^{D B}\right)\right), \quad m \mapsto\left\{(A, B) \in \mathfrak{P}^{c o}\left(\mathbb{K}^{D B}\right) \mid m \in B\right\}
\end{gathered}
$$

define an isomorphism between topological contexts.

We conclude our considerations with the following Representation Theorem:

Theorem 6.16 (Representation Theorem). For every regular double Boolean algebra $\underline{D}$ the context $\mathbb{K}^{D B}(\underline{D})$ is a standard topological context with $\underline{\mathfrak{P}}^{\text {co }}\left(\mathbb{K}^{D B}(\underline{D})\right) \simeq \underline{D}$. For every standard topological context $\mathbb{K}^{D B}, \mathfrak{P}^{c o}\left(\mathbb{K}^{D B}\right)$ is a regular double Boolean algebra and $\mathbb{K}^{D B} \simeq \mathbb{K}^{D B}\left(\mathfrak{P}^{c o}\left(\mathbb{K}^{D B}\right)\right)$. Moreover, the set of all clopen concepts of the standard DB-topological context of every regular double Boolean algebra $\underline{D}$ is isomorphic to $\underline{D}_{\sqcap} \cap \underline{D}_{\sqcup}$.

This representation can easily be extended to a categorical duality, with morphisms appropriately chosen. The methods used for this duality are widely described in [1]. 


\section{References}

[1] Breckner, B.E., Săcărea, C., Some Categorical Aspects in Topological Formal Concept Analysis, Mathematica, 59(2017), no. 1-2, 15-31.

[2] Ganter, B., Wille, R., Formal Concept Analysis - Mathematical Foundations, Springer, 1999.

[3] Hartung, G., A topological representation of lattices, Algebra Universalis, 29 (1992), 273-299.

[4] Hartung, G., An Extended Duality for Lattices, General Algebra and Applications, Heldermann-Verlag, Berlin, 1993, 126-142.

[5] Hartung, G., Kamara, M., Săcărea, C., A topological representation for polarity lattices, Acta Math. Univ. Comenian. (N.S.), 68(1999), no. 1, 49-70.

[6] Herrmann, Ch., Luksch, P., Skorsky, M., Wille, R., Algebras of Semiconcepts and Double Boolean Algebras in Contributions to General Algebra, 13, Verlag Johannes Heyn, Wien, 2000.

[7] Priestley, H.A., Representation of distributive lattices by means of ordered Stone spaces, Bull. Lond. Math. Soc., 2(1970), 186-190.

[8] Săcărea, C., Contextual Uniformities, Proceedings of the 11th International Conference on Formal Concept Analysis, LNAI 7880, Springer Verlag, Heidelberg, 2013, 244-253.

[9] Steen, L., Seebach jr., J.A., Counterexamples in Topology, Holt, Rinehart and Winston Inc., 1970.

[10] Stone, H., Topological representations of distributive lattices and Brouwerian logics, Časopis Pešt. Mat., 67(1937), 1-25.

[11] Urquhart, A., A topological representation theory for lattices, Algebra Universalis, 8(1978), 45-58.

[12] Wille, R., Restructuring lattice theory: an approach based on hierarchies of concepts, in I. Rival (ed.), Ordered Sets, Reidel, Dordrecht (vol. 83 of NATO Advanced Studies Institute), Boston, 1982, 445-470.

[13] Wille, R., Boolean Concept Logic, in Proceedings of the 8th International Conference on Conceptual Structures ICCS 2000, Lecture Notes in Computer Science, 1867, Springer, 317-331.

[14] Wille, R., Boolean Judgement Logic, in Proceedings of the 9th International Conference on Conceptual Structures ICCS 2001, Lecture Notes in Computer Science, 2120, Springer, 115-128.

Brigitte E. Breckner

Babeş-Bolyai University

Faculty of Mathematics and Computer Sciences

1, Kogălniceanu Street, 400084 Cluj-Napoca, Romania

e-mail: brigitte@math.ubbcluj.ro

Christian Săcărea

Babeş-Bolyai University

Faculty of Mathematics and Computer Sciences

1, Kogălniceanu Street, 400084 Cluj-Napoca, Romania

e-mail: csacarea@math.ubbcluj.ro 